\title{
Multi-copy and stochastic transformation of multipartite pure states
}

\author{
Lin Chen ${ }^{1, * \text { * }}$ and Masahito Hayashi ${ }^{2,1}$, 由 \\ ${ }^{1}$ Centre for Quantum Technologies, National University of Singapore, 3 Science Drive 2, 117542, Singapore \\ ${ }^{2}$ Graduate School of Information Sciences, Tohoku University, Aoba-ku, Sendai, 980-8579, Japan
}

(Dated: November 9, 2018)

\begin{abstract}
Characterizing the transformation and classification of multipartite entangled states is a basic problem in quantum information. We study the problem under two most common environments, local operations and classical communications (LOCC), stochastic LOCC and two more general environments, multi-copy LOCC (MCLOCC) and multi-copy SLOCC (MCSLOCC). We show that two transformable multipartite states under LOCC or SLOCC are also transformable under MCLOCC and MCSLOCC. What's more, these two environments are equivalent in the sense that two transformable states under MCLOCC are also transformable under MCSLOCC, and vice versa. Based on these environments we classify the multipartite pure states into a few inequivalent sets and orbits, between which we build the partial order to decide their transformation. In particular, we investigate the structure of SLOCC-equivalent states in terms of tensor rank, which is known as the generalized Schmidt rank. Given the tensor rank, we show that GHZ states can be used to generate all states with a smaller or equivalent tensor rank under SLOCC, and all reduced separable states with a cardinality smaller or equivalent than the tensor rank under LOCC. Using these concepts, we extended the concept of "maximally entangled state" in the multi-partite system.
\end{abstract}

PACS numbers: 03.65.Ud, 03.67.Mn, 03.67.-a

\section{INTRODUCTION}

One of the main tasks in quantum information theory is to find out how many different ways there exist, in which several spatially distributed objects could be entangled under certain prior environments with restricted physical resource. For example, we often use local operations assisted with classical communication (LOCC) with which one can obtain quantum resources definitely. This is a key condition to transform and prepare different multipartite states from each other, which are the basic ingredient in quantum-information tasks, such as GHZ states in quantum teleportation [1] and graph states in quantum computation [2]. It is known that bipartite pure entangled states are interconvertible in the asymptotic LOCC transformations [3]. In other words, all bipartite pure entangled states can be used to perform the same quantum-information task in the asymptotic regime.

On the other hand, the problem becomes complex for multipartite states. For example, it is known that two pure states are transformable from each other with certainty, namely equivalent if and only if they are related by local unitary operation (LU), i.e., $|\psi\rangle=\bigotimes_{i} U_{i}|\varphi\rangle$ where $U_{i}$ are unitary operators [4]. However in this way we can remove only very few parameters that characterize the system. As a result, we have to compare quantum states with an exponentially increasing number of parameters and it is thus unlikely to get a clear judgement of their convertibility under LOCC. To reduce the difficulty, Bennett et al [4] introduced another environ-

*Electronic address: cqtcl@nus.edu.sg (Corresponding ${ }^{\sim}$ Author)

${ }^{\dagger}$ Electronic address: hayashi@math.is.tohoku.ac.jp ment, namely the conversion of states through stochastic LOCC (SLOCC) with a nonzero success probability. Two pure states of a multipartite system are equivalent under SLOCC if and only if they are related by an invertible local operator, i.e., $|\psi\rangle=\bigotimes_{i} A_{i}|\varphi\rangle$ where $A_{i}$ are invertible operators [5]. For instance, concerning the three-qubit fully entangled states which contains no factorized systems under SLOCC, there exist only two different families namely the Greenberger-Horne-Zeilinger (GHZ) state [6] and the $W$ state [5]. In principle, the total classification of $2 \times M \times N$ states under SLOCC is realizable in terms of hyper-determinant [7], range criterion [8] and matrix pencil [9]. Furthermore, with SLOCC researchers have also addressed the behavior of multiqubit states, which are the fundamental resource in quantum computation and communications. In addition, the SLOCC environment is also a key technique to a few other important quantum information tasks, such as entanglement distillation [10].

In this paper we generalize the above two environments, which focus on one-copy transformation of quantum states. We propose the multi-copy LOCC (MCLOCC) and multi-copy SLOCC (MCSLOCC) in the sense that one can use many copies of states to produce a target state under LOCC and SLOCC, respectively 11. In fact, when a state $|\varphi\rangle$ can be transformed to $|\psi\rangle$ by SLOCC, we need to prepare many copies of $|\varphi\rangle$ for generating the state $|\psi\rangle$. So, from the operational viewpoint, it is natural to consider the convertibility from plural copies of the given state $|\varphi\rangle$ For example, the transformation between bipartite pure states is subjective to Nielsen's majorization theorem [12], and states violating the theorem are usually not inter-convertible. However as already mentioned [3], we can produce any target state with enough copy of input states. The fact 
indicates that there is an essential difference between onecopy and multi-copy transformation. This is important for various quantum-information tasks which may require different kinds of states as the input states. By studying MCLOCC and MCSLOCC, we can more effectively make use of the quantum resources. In particular, we will show that the two environments are equivalent in the sense that two transformable states under MCLOCC are also transformable under MCSLOCC, and vice versa. This is different from the relation between LOCC and SLOCC environment, where two equivalent states under SLOCC are often inequivalent under LOCC.

Based on LOCC, SLOCC and MCLOCC environments, we build a hierarchy to classify the multipartite pure states. We show that two transformable states under LOCC are also transformable under SLOCC, and further transformable under MCLOCC. In this sense, the LOCC environment is superior to other two environments. To characterize the transformation under SLOCC, we introduce the concept of tensor rank, which is also known as the Schmidt measure of entanglement [13]. As tensor rank is an invariant under invertible SLOCC, in terms of it and the local ranks of reduced density operators we can further characterize the structure of multipartite states under SLOCC.

We also propose the concept of maximally entangled states in under a given environment, in the sense that it can be used to generate all states in a set or orbit. First, we will show that any fully entangled $n$-partite pure state is maximal concerning MCLOCC among all $n$-partite pure states. Furthermore, for a given tensor rank, any state SLOCC-equivalent to GHZ states is a maximally entangled state concerning SLOCC in the set of states with the tensor rank. Third, we introduce the concept cardinality to describe the smallest number of product states forming the convex sum of a separable state, whose purification we name as the reduced separable pure states. Then we show that the GHZ state with rank $d$ is maximal concerning LOCC among reduced separable pure states with the largest cardinality $d$.

The rest of the paper is organized as follows. In Sec. II we define the basic environment for state transformation including multi-copy LOCC (MCLOCC), SLOCC and LOCC. We also establish their relations such as partial order and equivalence. In Sec. III, IV and V we classify the multipartite pure states by three elementary steps in terms of MCLOCC, SLOCC and LOCC, respectively. We conclude in Sec. VI.

\section{DEFINITIONS AND GENERAL CLASSIFICATION}

We start by defining a few useful criterion for one-way transformation between multipartite pure states.

Definition 1 We denote $\left|\psi_{1}\right\rangle \succ_{L O C C}\left|\psi_{2}\right\rangle$ when there exists LOCC that transforms $\left|\psi_{1}\right\rangle$ to $\left|\psi_{2}\right\rangle$. We denote $\left|\psi_{1}\right\rangle \succ_{S L O C C}\left|\psi_{2}\right\rangle$ when there exists matrices $A_{1}, \ldots, A_{n}$ such that $A_{1} \otimes \ldots \otimes A_{n}\left|\psi_{1}\right\rangle=\left|\psi_{2}\right\rangle$.

Definition 2 We denote $\left|\psi_{1}\right\rangle \succ_{M C L O C C}\left|\psi_{2}\right\rangle$ (multicopy LOCC) when there exists integer $n$ such that $\left|\psi_{1}\right\rangle^{\otimes n} \succ_{L O C C}\left|\psi_{2}\right\rangle$. We denote $\left|\psi_{1}\right\rangle \succ_{M C S L O C C}\left|\psi_{2}\right\rangle$ (multi-copy SLOCC) when there exists integer $n$ such that $\left|\psi_{1}\right\rangle^{\otimes n} \succ_{S L O C C}\left|\psi_{2}\right\rangle$.

Trivially, when $\left|\psi_{1}\right\rangle \quad \succ_{\text {LOCC }}\left|\psi_{2}\right\rangle$, we have $\left|\psi_{1}\right\rangle \succ_{S L O C C}\left|\psi_{2}\right\rangle$. Although the converse does not generally hold (e.g., $\cos \theta|00\rangle+\sin \theta|11\rangle \succ_{S L O C C}$ $(|00\rangle+|11\rangle) / \sqrt{2}$ but the transformation $\cos \theta|00\rangle+$ $\sin \theta|11\rangle \succ_{\text {LOCC }}(|00\rangle+|11\rangle) / \sqrt{2}$ is prohibited by the majorization criterion [12]), we have a further result that when $\left|\psi_{1}\right\rangle \succ_{S L O C C}\left|\psi_{2}\right\rangle,\left|\psi_{1}\right\rangle \succ_{M C L O C C}\left|\psi_{2}\right\rangle$. This is because when there exists POVM elements $A_{1}, \cdots, A_{n}$ which can be used to do the transformation $A_{1} \otimes \ldots \otimes$ $A_{n}\left|\psi_{1}\right\rangle=\left|\psi_{2}\right\rangle$ with nonzero probability $p>0$. Then we can repeat the measurement many times until we succeed with the probability $1-(1-p)^{n}, n \rightarrow \infty$. In this sense, we can deterministically transform the state $\left|\psi_{1}\right\rangle$ into $\left|\psi_{2}\right\rangle$ with sufficiently large $n$.

However, the converse does not hold in general. That is, there are states $\left|\psi_{1}\right\rangle,\left|\psi_{2}\right\rangle$ such that when $\left|\psi_{1}\right\rangle \succ_{M C L O C C}\left|\psi_{2}\right\rangle$, but not $\left|\psi_{1}\right\rangle \succ_{S L O C C}\left|\psi_{2}\right\rangle$. A typical example is the tripartite GHZ and W state

$$
\begin{aligned}
|\mathrm{GHZ}\rangle & =\frac{1}{\sqrt{2}}(|000\rangle+|111\rangle), \\
|\mathrm{W}\rangle & =\frac{1}{\sqrt{3}}(|001\rangle+|010\rangle+|100\rangle),
\end{aligned}
$$

which are known to be inequivalent under reversible SLOCC [5]. However, it is a necessary and sufficient condition that two-copy GHZ states are able to generate one W state under LOCC [14]. That is,

$$
|\mathrm{GHZ}\rangle^{\otimes 2} \succ_{L O C C}|\mathrm{~W}\rangle \text {. }
$$

Next, we can characterize the relation between the environment of MCLOCC and MCSLOCC. By using the same argument for deriving $\left|\psi_{1}\right\rangle \succ_{S L O C C}$ $\left|\psi_{2}\right\rangle \Rightarrow\left|\psi_{1}\right\rangle \quad \succ_{M C L O C C}\left|\psi_{2}\right\rangle$, we can get $\left|\psi_{1}\right\rangle \succ_{M C S L O C C}\left|\psi_{2}\right\rangle \Rightarrow\left|\psi_{1}\right\rangle \succ_{M C L O C C}\left|\psi_{2}\right\rangle$. On the other hand, suppose we have $\left|\psi_{1}\right\rangle \succ_{M C L O C C}\left|\psi_{2}\right\rangle$. Generally we can suppose $\left|\psi_{1}\right\rangle=\bigotimes_{i}\left|\varphi_{i 1}\right\rangle,\left|\varphi_{i 1}\right\rangle \in$ $\mathcal{H}_{i}$ are fully entangled. According to the definition of $M C L O C C$, we can get the expression $\left|\psi_{2}\right\rangle=$ $\bigotimes_{i}\left|\varphi_{i 2}\right\rangle,\left|\varphi_{i 2}\right\rangle \in \mathcal{H}_{i}$. In other words we have

$$
\left|\psi_{1}\right\rangle \succ_{M C L O C C}\left|\psi_{2}\right\rangle \Rightarrow\left|\varphi_{i 1}\right\rangle \succ_{M C L O C C}\left|\varphi_{i 2}\right\rangle
$$

. Let $\mathcal{H}_{i}=\bigotimes_{j=1}^{n_{i}} \mathcal{H}_{i, j}$, we can use the states $\left|\varphi_{i 1}\right\rangle$ to get bell states in the bipartite space $\mathcal{H}_{i, 1} \otimes \mathcal{H}_{i, j}, j=2, \cdots, n_{i}$ under SLOCC. So we can use the bell states to generate an arbitrary state $\left|\varphi_{i 2}\right\rangle$ by teleportation. Hence, we get

$$
\begin{aligned}
\left|\varphi_{i 1}\right\rangle \succ_{M C L O C C}\left|\varphi_{i 2}\right\rangle & \Rightarrow\left|\varphi_{i 1}\right\rangle \succ_{M C S L O C C}\left|\varphi_{i 2}\right\rangle \\
& \Rightarrow\left|\psi_{1}\right\rangle \succ_{M C S L O C C}\left|\psi_{2}\right\rangle .
\end{aligned}
$$

As a short summary of the above arguments, we have 
Theorem 3 For two n-partite pure states $\left|\psi_{1}\right\rangle$ and $\left|\psi_{2}\right\rangle$, we have

$$
\begin{array}{ccc}
\left|\psi_{1}\right\rangle & \succ_{\text {LOCC }} & \left|\psi_{2}\right\rangle \\
& \Downarrow & \\
\left|\psi_{1}\right\rangle & \succ_{\text {SLOCC }} & \left|\psi_{2}\right\rangle \\
& \Downarrow & \\
\left|\psi_{1}\right\rangle & \succ_{M C L O C C} & \left|\psi_{2}\right\rangle \\
& \Uparrow & \\
\left|\psi_{1}\right\rangle & \succ_{M C S L O C C} & \left|\psi_{2}\right\rangle .
\end{array}
$$

It is noticeable that in the four definitions of Theorem 3, only the definition $\left|\psi_{1}\right\rangle \succ_{M C L O C C}\left|\psi_{2}\right\rangle$ is asymptotic while other three are deterministic. In other words, under MCLOCC we obtain a state $\rho$ such that the fidelity $F\left(\rho,\left|\psi_{2}\right\rangle\right) \simeq 1$ when the number of copies $n \rightarrow \infty$. However in all other three definitions, we are required to get an exact state $\left|\psi_{2}\right\rangle$ from $\left|\psi_{1}\right\rangle^{\otimes n}$ with a finite $n$.

Because of Theorem 3 we will use the definition $\left|\psi_{1}\right\rangle \succ_{M C L O C C}\left|\psi_{2}\right\rangle$ which is equal to $\left|\psi_{1}\right\rangle \succ_{M C S L O C C}$ $\left|\psi_{2}\right\rangle$ from now on. Based on the results of one-way transformation, we study the relations under two-way (invertible) transformation.

Definition 4 We denote $\left|\psi_{1}\right\rangle \cong_{M C L O C C}\left|\psi_{2}\right\rangle$, when $\left|\psi_{1}\right\rangle \succ_{M C L O C C}\left|\psi_{2}\right\rangle$ and $\left|\psi_{2}\right\rangle \succ_{M C L O C C}\left|\psi_{1}\right\rangle$. So $\left|\psi_{1}\right\rangle$ is called MCLOCC-equivalent to $\left|\psi_{2}\right\rangle$. We denote the set of states $\left|\psi_{2}\right\rangle$ by $\mathcal{O}_{M C L O C C}\left(\left|\psi_{1}\right\rangle\right)$, namely the MCLOCC orbit of state $\left|\psi_{1}\right\rangle$.

Definition 5 Similarly, we denote $\left|\psi_{1}\right\rangle \cong_{S L O C C}\left|\psi_{2}\right\rangle$. So $\left|\psi_{1}\right\rangle$ is called SLOCC-equivalent to $\left|\psi_{2}\right\rangle$. We denote as the $S L O C C$ orbit $\mathcal{O}_{S L O C C}\left(\left|\psi_{2}\right\rangle\right)$, which is known to consist of states $\left|\psi_{1}\right\rangle=\bigotimes_{i} A_{i}\left|\psi_{2}\right\rangle$ where $A_{i}$ are invertible [5].

Definition 6 Similarly, we denote $\left|\psi_{1}\right\rangle \cong_{L O C C}\left|\psi_{2}\right\rangle$. So $\left|\psi_{1}\right\rangle$ is called LOCC-equivalent to $\left|\psi_{2}\right\rangle$. We denote as the LOCC orbit $\mathcal{O}_{\text {LOCC }}\left(\left|\psi_{2}\right\rangle\right)$, which is known to consist of states $\left|\psi_{1}\right\rangle=\bigotimes_{i} U_{i}\left|\psi_{2}\right\rangle$ where $U_{i}$ are unitary [4].

Based on these definitions, we can generalize Theorem 3 to the two-way (invertible) case.

Theorem 7 For two n-partite pure states $\left|\psi_{1}\right\rangle$ and $\left|\psi_{2}\right\rangle$, we have

$$
\begin{array}{rlr}
\left|\psi_{1}\right\rangle & \cong_{L O C C} \quad\left|\psi_{2}\right\rangle \\
& \Downarrow \\
\left|\psi_{1}\right\rangle & \cong_{\text {SLOCC }} \quad\left|\psi_{2}\right\rangle \\
& \Downarrow \\
\left|\psi_{1}\right\rangle \cong_{M C L O C C} & \left|\psi_{2}\right\rangle \\
& \multirow{2}{}{} & \\
\left|\psi_{1}\right\rangle & \cong_{M C S L O C C} & \left|\psi_{2}\right\rangle .
\end{array}
$$

Similarly, we will use the definition $\left|\psi_{1}\right\rangle \cong_{M C L O C C}$ $\left|\psi_{2}\right\rangle$ which is equal to $\left|\psi_{1}\right\rangle \cong_{M C S L O C C}\left|\psi_{2}\right\rangle$ from now on. We say that two states/orbits are $X$ incomparable, namely $\left|\psi_{1}\right\rangle \varlimsup_{X}\left|\psi_{2}\right\rangle$ when the partial order $\succ_{X}$ does not hold for both directions.

\section{CLASSIFICATION OF MULTIPARTITE PURE STATES UNDER MCLOCC}

To show the power of definitions for multipartite pure states in last section, we classify the multipartite pure states in terms of Theorem 7 . Following the three inclusion relations there, we use three corresponding elementary steps as our strategy. That is,

1. First, we divide multipartite pure states into a few inequivalent MCLOCC orbits and clarify the three partial orders under MCLOCC, SLOCC, and LOCC between these orbits.

2. Second, we divide each MCLOCC orbit into a few inequivalent SLOCC sets, orbits and clarify their partial order under SLOCC.

3. Third, we give a few examples from the SLOCC sets and orbits and study their partial order under LOCC.

These steps will be carried out in Sec. III, IV and V respectively.

Let us carry out elementary step 1 which is relatively easy to finish. For this purpose, we define the concept of the independence for a given pure state $|\psi\rangle$ on the multipartite system $A_{1}, A_{2}, \ldots, A_{n}$. Two systems $A_{1}$ and $A_{2}$ are independent for a pure multipartite state $|\psi\rangle$ when the density matrix on the composite system $A$ and $B$ has the form $\rho_{A} \otimes \rho_{B}$. In particular, the two systems $A_{1}$ and $A_{2}$ are completely independent for $|\psi\rangle$ when the two systems $A_{1}$ and $A_{2}$ are independent for any state in the orbit $\mathcal{O}_{S L O C C}(|\psi\rangle)$. Two parties $A_{1}$ and $A_{2}$ are completely independent for $|\psi\rangle$ if and only if there are two distinct groups $\left\{A_{a_{1}}, \ldots, A_{a_{k}}\right\}$ and $\left\{A_{b_{1}}, \ldots, A_{b_{l}}\right\}$ among $A_{3}, \ldots, A_{n}$ and two pure states $\left|\psi_{1}\right\rangle$ on $A_{1}, A_{a_{1}}, \ldots, A_{a_{k}}$ and $\left|\psi_{2}\right\rangle$ on $A_{2}, A_{b_{1}}, \ldots, A_{b_{l}}$ such that $|\psi\rangle=\left|\psi_{1}\right\rangle \otimes\left|\psi_{2}\right\rangle$. Using this relation, we can find that two systems $A_{1}$ and $A_{2}$ are completely independent for a state $|\psi\rangle$ if and only if two systems $A_{1}$ and $A_{2}$ are completely independent for the state $|\psi\rangle^{\otimes n}$.

For a given multipartite state $|\psi\rangle$, we define a graph to connect two parties that are not independent. We denote such a graph by $G(|\psi\rangle)$. For example, when $|\psi\rangle$ is $|0000\rangle+|0110\rangle+|1200\rangle+|1310\rangle+|0001\rangle+|0111\rangle+$ $|1201\rangle+|1311\rangle$, the party $A_{1}$ is independent of the parties $A_{3}$ and $A_{4}$, the party $A_{2}$ is independent of the party $A_{4}$, the party $A_{3}$ is independent of the parties $A_{1}$ and $A_{4}$, and the party $A_{4}$ is independent of the parties $A_{1}, A_{2}$, and $A_{3}$. However, the parties $A_{1}$ and $A_{3}$ are not completely independent. We describe the graph $G(|\psi\rangle)$ explicitly in Fig. 11. 


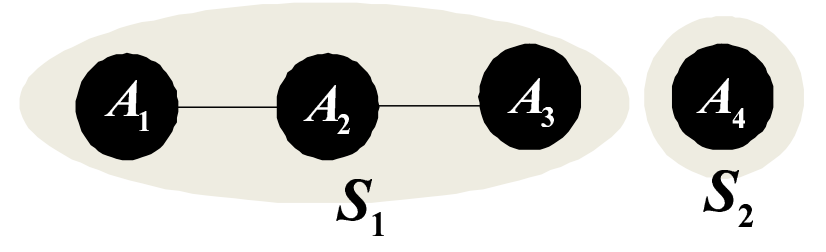

FIG. 1: Graph $G(|\psi\rangle)$ when $|\psi\rangle$ is $|0000\rangle+|0110\rangle+|1200\rangle+$ $|1310\rangle+|0001\rangle+|0111\rangle+|1201\rangle+|1311\rangle$.

Using this graph, we obtain a partition of the parties $A_{1}, \ldots, A_{n}$ and denote it by $\left\{S_{1}(|\psi\rangle), \ldots, S_{k}(|\psi\rangle)\right\}$. That is, $S_{1}(|\psi\rangle), \ldots, S_{k}(|\psi\rangle)$ are subsets of $\left\{A_{1}, \ldots, A_{n}\right\}$ and $\cup_{i=1}^{k} S_{i}(|\psi\rangle)=\left\{A_{1}, \ldots, A_{n}\right\}$. Besides, $S_{i}(|\psi\rangle) \cap S_{j}(|\psi\rangle)$ is empty when $i \neq j$. For any two parties $A_{1}$ and $A_{2}$ in $S_{i}(|\psi\rangle), A_{1}$ connects $A_{2}$ in the graph $G(|\psi\rangle)$, or there exist parties $A_{c_{1}}, \ldots, A_{c_{l}}$ in $S_{i}(|\psi\rangle)$ such that $A_{1}$ connects $A_{c_{1}}, A_{2}$ connects $A_{c_{l}}$, and $A_{c_{i}}$ connects $A_{c_{i+1}}$ in the graph $G(|\psi\rangle)$. In the former case, we call $A_{1}$ connects $A_{2}$ in the graph $G(|\psi\rangle)$ directly while in the later case, we call $A_{1}$ connects $A_{2}$ in the graph $G(|\psi\rangle)$ indirectly. So, in other words, all elements of $S_{i}(|\psi\rangle)$ are connected with each other at least indirectly. Further, any element of $S_{i}(|\psi\rangle)$ is not connected with any element of $S_{j}(|\psi\rangle)$ even indirectly when $i \neq j$.

In order to characterize the partition of the set $\left\{A_{1}, \ldots, A_{n}\right\}$, we define the partial order among partitions. For two given partitions of the set $\left\{A_{1}, \ldots, A_{n}\right\}$, $\left\{S_{1}, \ldots, S_{k}\right\}$ and $\left\{S_{1}^{\prime}, \ldots, S_{l}^{\prime}\right\}$, we denote $\left\{S_{1}, \ldots, S_{k}\right\} \succ$ $\left\{S_{1}^{\prime}, \ldots, S_{l}^{\prime}\right\}$ when any subset $S_{i}^{\prime}$ in $\left\{S_{1}^{\prime}, \ldots, S_{l}^{\prime}\right\}$ belongs to a subset $S_{i}$ in $\left\{S_{1}, \ldots, S_{k}\right\}$. That is, the partition $\left\{S_{1}^{\prime}, \ldots, S_{l}^{\prime}\right\}$ is smaller than the partition $\left\{S_{1}, \ldots, S_{k}\right\}$.

Since the pure state $|\psi\rangle$ has the form $\bigotimes_{i}\left|\psi_{i}\right\rangle_{S_{i}(|\psi\rangle)}$, we can classify multipartite states by using the partition of the set $\left\{A_{1}, \ldots, A_{n}\right\}$.

$\mathcal{O}\left(S_{1}, \ldots, S_{k}\right):=\left\{|\psi\rangle \mid\left\{S_{1}(|\psi\rangle), \ldots, S_{k}(|\psi\rangle)\right\}=\left\{S_{1}, \ldots, S_{k}\right\}\right\}$.

From the above discussion, we can show the "only if" part of the following theorem.

Theorem 8 Any state in $\mathcal{O}\left(S_{1}^{\prime}, \ldots, S_{l}^{\prime}\right)$ can be generated by MCLOCC from any state of $\mathcal{O}\left(S_{1}, \ldots, S_{k}\right)$ if and only if the relation $\left\{S_{1}, \ldots, S_{k}\right\} \succ\left\{S_{1}^{\prime}, \ldots, S_{l}^{\prime}\right\}$ holds.

The "if" part can be shown by the following lemmas. Using the above theorem, for any partition $\left\{S_{1}, \ldots, S_{l}\right\}$ of the set $\left\{A_{1}, \ldots, A_{n}\right\}$, the set $\mathcal{O}\left(S_{1}, \ldots, S_{l}\right)$ is an MCLOCC equivalent class and characterized as follows.

$$
\mathcal{O}\left(S_{1}, \ldots, S_{l}\right)=\mathcal{O}\left(S_{1}\right) \otimes \ldots \otimes \mathcal{O}\left(S_{l}\right) .
$$

In fact, for $S_{1}=\left\{A_{n_{1}}, \ldots, A_{n_{l}}\right\}$, the set $\mathcal{O}\left(S_{1}\right)$ is equal to the MCLOCC equivalent class generated by $\left|\mathrm{GHZ}_{d: A_{n_{1}}, \ldots, A_{n_{l}}}\right\rangle:=\sum_{i=1}^{d}|i \ldots i\rangle_{A_{n_{1}}, \ldots, A_{n_{l}}}$, where $d \geq$ 2. Hence, in the multipartite system $A_{1}, \ldots, A_{n}$, the state $\left|\mathrm{GHZ}_{d: A_{1}, \ldots, A_{n}}\right\rangle$ is maximal in the sense of MCLOCC.
Now, we prepare lemmas for our proof of "if" part of Theorem 8

Lemma 9 When the tensor rank of the state pure state $|\psi\rangle$ on $A_{1}, \ldots, A_{n}$ is $d$, it can be generated by $S L O C C$ from the GHZ state $\left|\mathrm{GHZ}_{d: A_{1}, \ldots, A_{n}}\right\rangle$.

Proof. There exist $n$ sets of non-normalized vectors $\left\{\left|a_{1, i}\right\rangle\right\}_{i=1}^{d}, \cdots,\left\{\left|a_{n, i}\right\rangle\right\}_{i=1}^{d}$ such that $|\psi\rangle=\sum_{i=1}^{d}\left|a_{1, i}\right\rangle \otimes$ $\cdots \otimes\left|a_{n, i}\right\rangle$. So we can find the local operators $A_{i}, i=$ $1, \cdots, n$ such that $\bigotimes_{i=1}^{n} A_{i} \sum_{i=1}^{d}|i \cdots i\rangle=|\psi\rangle$.

Using this lemma, for an arbitrary subset $S_{i} \subset$ $\left\{A_{1}, \ldots, A_{n}\right\}$, we can show that any state in $\mathcal{O}\left(S_{i}\right)$ can be generated by MCLOCC from the GHZ state of $S_{i}$. So, it is sufficient to show that the GHZ state of $S_{i}$ can be generated by MCLOCC from any state in $\mathcal{O}\left(S_{i}\right)$. In order to prove this argument, we prepare the following two lemmas.

Lemma 10 For given $m$ parties $A_{1}, \ldots, A_{m}$ and an integer $1 \leq n \leq m-1$, we denote the composite system $A_{n}$ and $A_{n+1}$ by $B$. Then, the state $\left|\mathrm{GHZ}_{d: A_{1}, \ldots, A_{n-1}, B, A_{n+2}, \ldots, A_{m}}\right\rangle$ can be generated from $\left|\mathrm{GHZ}_{d: A_{1}, \ldots, A_{n}}\right\rangle \otimes\left|\mathrm{GHZ}_{d: A_{n+1}, \ldots, A_{m}}\right\rangle$ by LOCC concerning $A_{1}, \ldots, A_{n-1}, B, A_{n+2}, \ldots, A_{m}$.

Proof. Let $P_{i}$ be the projection to the subspace spanned by $|1\rangle|1+i\rangle, \ldots,|d\rangle|d+i\rangle$, where $d+i \equiv i($ $\bmod d$ ). First, we apply the measurement $\left\{P_{i}\right\}_{i=1}^{d}$ on the system $B$, which is the composite system $A_{n}$ and $A_{n+1}$. When we obtain the outcome $i$, we apply the local unitary $|j\rangle \rightarrow|j-i\rangle$ on the systems $A_{n+2}, \ldots, A_{m}$. Then, we obtain the GHZ state on $A_{1}, \ldots, A_{n-1}, B, A_{n+2}, \ldots, A_{m}$.

Lemma 11 When $A_{1}$ and $A_{2}$ are not independent for a pure multipartite state $|\psi\rangle,\left|\Psi_{2: A_{1}, A_{2}}\right\rangle:=\sum_{i=1}^{2}|i i\rangle_{A_{1}, A_{2}}$ can be generated by SLOCC from $|\psi\rangle$. Hence, when $A_{1}$ and $A_{2}$ are not completely independent for a pure multipartite state $|\psi\rangle,\left|\Psi_{2: A_{1}, A_{2}}\right\rangle$ can be generated by SLOCC from $|\psi\rangle$.

Proof. For any $i \neq 1,2$, there is a rank-one projection $P$ on the system $A_{i}$ such that $A_{1}$ and $A_{2}$ are not independent for a pure multipartite state $A_{i}|\psi\rangle$. Thus, the state $|\psi\rangle$ can be converted to a pure entangled state on $A_{1}$ and $A_{2}$ by SLOCC. Using this state, we can generate $\left|\psi_{2: A_{1}, A_{2}}\right\rangle$ by SLOCC.

We choose an arbitrary pure state $|\psi\rangle$ in $\mathcal{O}\left(S_{i}\right)$, where $S_{i}=\left\{A_{a_{1}}, \ldots, A_{a_{k}}\right\}$. Using Lemma 11, we can produce Bell states between two parties connected in the graph $G(|\psi\rangle)$ by MCLOCC from $|\psi\rangle$. Then, using Lemma 10 ,

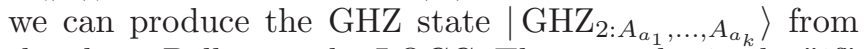
the above Bell states by LOCC. Thus, we obtain the "if" part of Theorem 8 . 


\section{CLASSIFICATION OF MULTIPARTITE PURE STATES UNDER SLOCC}

In this section we study elementary step 2. Due to Eq. (6), it is sufficient to consider the orbit decomposition of the set $\mathcal{O}\left(\left\{A_{1}, \ldots, A_{n}\right\}\right)$ by SLOCC.

The problem has been widely studied in the past decade [5, 8, 15 18] even in the multipartite scenario. In particular, it has been pointed out that deciding whether a GHZ state is SLOCC convertible into a multipartite state is equal to the derivation of tensor rank [15]. This is the generalization of the Schmidt rank in multipartite systems. Formally, for states in $N$-partite quantum systems, each of which is described by a $d$-dimensional Hilbert space $\eta_{i}(i=1, \ldots, N)$, the tensor $\operatorname{rank} \operatorname{rk}(\psi)$ of a state $|\psi\rangle \in \bigotimes_{\alpha=1}^{N} \eta_{\alpha}$, defined as the smallest number of product states $\left\{\bigotimes_{\alpha=1}^{N}\left|\phi_{i}^{\alpha}\right\rangle\right\}_{i=1 \ldots r k(\psi)}$ whose linear span contains $|\psi\rangle$. The tensor rank has been extensively studied in algebraic complexity theory [19, 20]. While it is easy to compute for $N=2$ (Schmidt rank), even for $N=3$, determining the rank of a state is NP-hard 21]. This is one reason why SLOCC convertibility in multipartite systems is so challenging.

It's well known that, classifying multipartite states in high dimensions is difficult for the number of parameters increases exponentionally with local ranks. Different from the traditional way, we will use tensor rank to make the problem more tractable. The classification with both tensor rank and local ranks provides a new perspective on the structure of multipartite states under SLOCC. In particular we will rely on the following result.

Lemma 12 Two SLOCC-equivalent pure states on the multipartite system $A_{1}, \ldots, A_{N}$ have to have identical SLOCC invariants, $\mathrm{rk}, r_{A_{1}}, \ldots, r_{A_{N}}$. So inequivalent SLOCC orbits have to have at least one nonidentical invariant out of $\mathrm{rk}, r_{A_{1}}, \ldots, r_{A_{N}}$. Besides, the $N+1$ SLOCC invariants are non-increasing under noninvertible $S L O C C$.

The lemma is obvious. Based on it, we classify multipartite pure states into a few inequivalent SLOCC orbits and build a few theorems to characterize them. First of all we divide the orbit $\mathcal{O}\left(\left\{A_{1}, \ldots, A_{n}\right\}\right)$ into a few principal SLOCC-closed sets in the light of only tensor rank

$$
\mathcal{O}\left(\left\{A_{1}, \ldots, A_{n}\right\}\right)=\bigcup_{d \geq 2} \mathcal{R}(d),
$$

where $\mathcal{R}(d)$ is the set of states with tensor rank $d$ in $\mathcal{O}\left(\left\{A_{1}, \ldots, A_{n}\right\}\right)$, which is closed under reversible SLOCC. By using Lemma 12, it suffices to consider only one principal SLOCC orbit, namely $\mathcal{R}(d)$ with constant $d \geq 2$. Taking into account other $N$ SLOCC invariants $r_{A_{1}}, \ldots, r_{A_{N}}$, we can split this SLOCC-closed set into $s u b$ SLOCC-closed sets denoted as

$$
\mathcal{R}(d)=\bigcup_{d \geq r_{A_{1}}, \ldots, r_{A_{N}}} \mathcal{R}\left(d \| r_{A_{1}}, \ldots, r_{A_{N}}\right),
$$

where $\mathcal{R}\left(d \| r_{A_{1}}, \ldots, r_{A_{N}}\right)$ is the set of states with constant tensor rank $d$ and local ranks $r_{A_{1}}, \ldots, r_{A_{N}}$. Evidently there are many states fulfilling the property. In this sense we can decompose the sub SLOCC-closed set into SLOCC orbits of SLOCC-inequivalent states

$\mathcal{R}\left(d \| r_{A_{1}}, \ldots, r_{A_{N}}\right)=\bigcup_{j} \mathcal{O}_{S L O C C}\left(\sum_{i=1}^{d}\left|a_{1, i}^{j}, \ldots, a_{N, i}^{j}\right\rangle\right)$,

where the ranks of the spaces spanned by $\left\{\left|a_{1, i}^{j}\right\rangle\right\}, \ldots,\left\{\left|a_{N, i}^{j}\right\rangle\right\}, \quad$ and $\quad\left\{\left|a_{1, i}^{j}, \ldots, a_{N, i}^{j}\right\rangle\right\} \quad$ are $r_{A_{1}}, \ldots, r_{A_{N}}$, and $d$, respectively. In particular, when $r_{A_{1}}$ is $d$, the states $\left\{\left|a_{1, i}^{j}\right\rangle\right\}$ can be restricted to the states $\{|i\rangle\}$. This fact can be applied to the cases of $r_{A_{j}}=d$.

By using the three steps in Eqs. 7, 8 and 9, we have given a clear hierarchy of fully entangled multipartite states. In each level states from different sets/orbits are SLOCC-inequivalent. From this hierarchy we see that the third level Eq. 9], namely splitting the sub SLOCCclosed set into SLOCC orbits is a key step to classify multipartite states in each principal SLOCC-closed set. This is the topic of subsection IVA.

\section{A. decomposition of sub SLOCC-closed sets}

We start by characterizing the simplest sub SLOCCclosed set in $\mathcal{R}(d)$. Note that we don't normalize the states because it doesn't influence the equivalence and partial order under SLOCC and LOCC.

Lemma 13 For any pure state $|\psi\rangle$ with $\operatorname{rk}(\psi)=$ $r_{A_{1}}(\psi)=\cdots=r_{A_{N}}(\psi)=d$, there exists invertible matrices $X_{1}, \ldots, X_{N}$ such that $X_{1} \otimes \cdots \otimes$ $X_{N} \sum_{i=1}^{d}|i \cdots i\rangle_{A_{1} \ldots A_{N}}=|\psi\rangle$. So we have

$$
\mathcal{R}(d \| d, d, d)=\mathcal{O}_{S L O C C}\left(\left|\mathrm{GHZ}_{d: A_{1} \ldots A_{N}}\right\rangle\right) .
$$

The d-dimensional GHZ state $\left|\mathrm{GHZ}_{\left.d: A_{1} \ldots A_{N}\right\rangle}\right\rangle$ is the canonical state of the orbit.

Proof. There exist $N$ sets of non-normalized linearly independent vectors $\left\{\left|a_{1, i}\right\rangle\right\}_{i=1}^{d}, \cdots,\left\{\left|a_{N, i}\right\rangle\right\}_{i=1}^{d}$ such that $|\psi\rangle=\sum_{i=1}^{d}\left|a_{1, i}\right\rangle \otimes \cdots \otimes\left|a_{N, i}\right\rangle$. So we can find the invertible local operators $A_{i}, i=1, \cdots, N$ such that $\bigotimes_{i=1}^{N} A_{i} \sum_{i=1}^{d}|i \cdots i\rangle=|\psi\rangle$.

However, other sub SLOCC-closed subsets are not SLOCC orbits of given states. To show this fact, we recall the classification of $2 \times 3 \times 3$ states under SLOCC [8].

Lemma 14 There are six inequivalent states in the $2 \times$ 


\section{$3 \times 3$ space under $S L O C C$}

$$
\begin{aligned}
& \left|\Psi_{1}\right\rangle=|000\rangle+|111\rangle+(|0\rangle+|1\rangle)|22\rangle, \quad \mathrm{rk}=3, \quad \\
& \left|\Psi_{2}\right\rangle=|010\rangle+|001\rangle+|112\rangle+|121\rangle, \quad \mathrm{rk}=4, \quad \\
& \left|\Psi_{3}\right\rangle=|000\rangle+|111\rangle+|022\rangle, \quad \mathrm{rk}=3, \quad \\
& \left|\Psi_{4}\right\rangle=|100\rangle+|010\rangle+|001\rangle+|112\rangle+|121\rangle, \quad \mathrm{rk}=4,
\end{aligned}
$$$$
\left|\Psi_{5}\right\rangle=|100\rangle+|010\rangle+|001\rangle+|022\rangle, \quad \mathrm{rk}=4,
$$

$\left|\Psi_{6}\right\rangle=|100\rangle+|010\rangle+|001\rangle+|122\rangle, \mathrm{rk}=4$.

So the $2 \times 3 \times 3$ state belongs to two principal SLOCCclosed sets $\mathcal{R}(4)$ and $\mathcal{R}(3)$. More precisely, they belong to only two sub SLOCC orbits respectively, i.e.,

$$
\begin{aligned}
& \mathcal{R}(3 \| 2,3,3)=\bigcup_{i=1,3} \mathcal{O}_{\text {SLOCC }}\left(\left|\Psi_{i}\right\rangle\right), \\
& \mathcal{R}(4 \| 2,3,3)=\bigcup_{i=2,4,5,6} \mathcal{O}_{\text {SLOCC }}\left(\left|\Psi_{i}\right\rangle\right) .
\end{aligned}
$$

By removing the parameters with locally invertible operators, we can generalize Eq. 17 to the $N$-partite states such as

$$
\begin{aligned}
\mathcal{R}(d \| d-1, d, \cdots, d) \\
=\bigcup_{j=1}^{d-1} \mathcal{O}_{S L O C C}\left(\sum_{i=1}^{d-1}|i\rangle^{\otimes N}+\sum_{i=1}^{j}|i\rangle|d\rangle^{\otimes(N-1)}\right) .
\end{aligned}
$$

Note that the $d-1$ states in the big parentheses are SLOCC-inequivalent. The reason is that they can be written as

$$
\begin{aligned}
\left|\varphi_{j}\right\rangle & =\sum_{i=1}^{j}|i\rangle\left(|i\rangle^{\otimes(N-1)}+|d\rangle^{\otimes(N-1)}\right)+\sum_{i=j+1}^{d-1}|i\rangle^{\otimes N} \\
& =\sum_{i=1}^{d-1}\left|a_{i, j}\right\rangle\left|\psi_{i, j}\right\rangle
\end{aligned}
$$

where $\left|a_{i, j}\right\rangle \in \mathcal{H}_{1}$ and $\left|\psi_{i, j}\right\rangle \in \bigotimes_{i=2}^{N} \mathcal{H}_{i}$. Because the state in the range of $\rho_{2, \cdots, N}$ has the expression $\sum_{i=1}^{j} a_{i}\left(|i\rangle^{\otimes(N-1)}+|d\rangle^{\otimes(N-1)}\right)+\sum_{i=j+1}^{d-1} a_{i}|i\rangle^{\otimes(N-1)}$, which is entangled if any coefficient $a_{i} \neq 0, i \in[1, j]$. Hence there are at least $j$ entangled states $\left|\psi_{i, j}\right\rangle$ in the state $\left|\varphi_{j}\right\rangle$. Because entanglement cannot be changed under locally invertible operators, the states $\left|\varphi_{j}\right\rangle$ cannot inter-convertible under SLOCC.

In general the decomposition of sub SLOCC-closed sets is not easy. As a partial result, we can use the method in [8, 9] to classify $2 \times M \times N$ states under SLOCC. Besides, tensor rank is also computable effectively for this family of states 9,22 . These results help carry out the hierarchy in Eq. 7.9] First by following Eq. 7 , we distribute the $2 \times M \times N$ states into the principal SLOCC-closed sets $\mathcal{R}(d)$. Next by following Eq. 8, we can decompose each $\mathcal{R}(d)$ into sub SLOCC-closed sets to which $2 \times M \times N$ states belong. Third we can decide

the SLOCC orbits in each sub SLOCC-closed sets, which realizes Eq. 9

One can similarly characterize $L \times M \times N(L \geq 3)$ pure states. However the problem becomes complex as the dimension increases, e.g., there are infinitely many inequivalent $2 \times 4 \times 4$ and $3 \times 3 \times 3$ states under SLOCC [8]. Actually it's been shown that the classification of tripartite pure states is a NP-hard problem [15]. In this context, it becomes quite important to figure out a general and clear configuration of the orbit $\mathcal{O}_{M C L O C C}^{A B C}$. This can be done through the SLOCC partial order between the principal and sub SLOCC-closed sets, which is the topic of next subsection.

\section{B. SLOCC partial order}

We have introduced three kinds of SLOCC classification for multipartite states in previous paragraphs, namely the principal SLOCC-closed set, the sub SLOCCclosed set, and the SLOCC orbit. To get a clear configuration of the orbit $\mathcal{O}\left(A_{1}, \ldots, A_{N}\right)$, we will investigate the orbits successively embedded into each other, i.e., $\mathcal{O}_{S L O C C}(|\psi\rangle) \subset \mathcal{R}\left(d \| r_{A_{1}}, \ldots, r_{A_{N}}\right) \subset \mathcal{R}(d) \subset$ $\mathcal{O}\left(A_{1}, \ldots, A_{N}\right)$. In other words, the former orbit or set always belong to the same latter orbit or set under SLOCC partial order, respectively. It will also help reduce the question of rapidly increasing parameters in high dimensions.

First, we study the partial order of different principal SLOCC-closed set, $\mathcal{R}\left(d_{1}\right)$ and $\mathcal{R}\left(d_{2}\right), d_{1}>d_{2}$. There are a few states in the former which can be converted into the latter via SLOCC. For example, we can readily realize $\left|\mathrm{GHZ}_{d_{1}: A_{1}, \ldots, A_{N}}\right\rangle \succ_{S L O C C}|\psi\rangle$ where $\forall|\psi\rangle \in \mathcal{R}\left(d_{2}\right)$. However for generic states, we cannot carry out the transformation as above. The biggest reason is that there are states in the SLOCC-closed set $\mathcal{R}\left(d_{1}\right)$, which have smaller local rank than that of some states in the SLOCC-closed set $\mathcal{R}\left(d_{2}\right)$, and local ranks cannot be increased under SLOCC as mentioned in Lemma 12 For example, the states $|111\rangle+|122\rangle+|213\rangle+|224\rangle$ and $\left|\mathrm{GHZ}_{3}\right\rangle$ have tensor rank four and three, respectively. However they are evidently SLOCC incomparable. On the other hand, the transformation $\mathcal{R}\left(d_{2}\right) \succ_{S L O C C}$ $\mathcal{R}\left(d_{1}\right)$ is forbidden due to the fact that tensor rank is non-increasing under SLOCC. So we conclude

Theorem 15 Generic states in different principal SLOCC-closed sets are SLOCC incomparable. That is, for $\left|\psi_{1}\right\rangle \in \mathcal{R}\left(d_{1}\right)$ and $\left|\psi_{2}\right\rangle \in \mathcal{R}\left(d_{2}\right), d_{1}>d_{2}$ we have $\left|\psi_{1}\right\rangle \varlimsup_{S L O C C}\left|\psi_{2}\right\rangle$. So we readily get the corollary that for generic states $\left|\psi_{1}\right\rangle \varlimsup_{L O C C}\left|\psi_{2}\right\rangle$.

Second, we study the partial order of different sub SLOCC-closed sets from the principal SLOCC-closed set $\mathcal{R}(d)$. We present the following result.

Theorem 16 The GHZ state with rank $d$ is maximal concerning SLOCC partial order among pure states with 
tensor rank d. That is, the GHZ state can be used to generate all states in the principal SLOCC-closed sets $\mathcal{R}(d)$ with some nonzero probability,

$$
\left|\mathrm{GHZ}_{d: A_{1}, \ldots, A_{N}}\right\rangle \succ_{S L O C C}|\psi\rangle, \quad \forall|\psi\rangle \in \mathcal{R}(d) .
$$

Proof. Similar to Lemma 13.

Third, we can easily indicate the partial order between SLOCC orbits $\mathcal{O}_{S L O C C}\left(\left|\psi_{i}\right\rangle\right)$ that form a sub SLOCC-closed set $\mathcal{R}\left(d \| r_{A_{1}}, \ldots, r_{A_{N}}\right)$. In the light of Lemma 12, two SLOCC-inequivalent states with identical four SLOCC invariants are SLOCC incomparable, and thus also LOCC incomparable. In last subsection, we have introduced the way to characterize SLOCCinequivalent states in $2 \times M \times N$ space. More efforts are still required for high dimensions.

As a short summary, in this section we have classified multipartite states based on Lemma 12 In our hierarchy of three levels, we decomposed the MCLOCC orbit $\mathcal{O}_{M C L O C C}^{A B C}$ into principal SLOCC-closed sets, from each of which we have proposed sub SLOCC-closed sets and decomposed them into inequivalent SLOCC orbits. We also have given the partial order under SLOCC. Our method is generally different from, and also greatly extends the previous methods of classifying multipartite states [8, 16].

In next section, we will extend our results to the LOCC classification and show the LOCC partial order between the orbits.

\section{LOCC PARTIAL ORDER}

In this section we carry out elementary step 3 for LOCC partial order. In the bipartite case, the state $\sum_{i=1}^{d}|i, i\rangle$ is called the maxially entangled state because the state $\sum_{i=1}^{d}|i, i\rangle$ is maximal concerning the LOCC partial order among pure bipartite states with rank $d$. Intuitively, we might expect that the state $\left|\mathrm{GHZ}_{d}\right\rangle$ can generate any pure state with tensor rank $d$ by LOCC in the multipartite system. However, it is not true even in the tripartite case with $\mathrm{rk}=r_{A}=r_{B}=r_{C}=2$, because the state $|0,0,0\rangle+(|0\rangle+|1\rangle)(|0\rangle+|1\rangle)(|0\rangle+|1\rangle)$ cannot be generated from the state $\left|\mathrm{GHZ}_{2}\right\rangle$ by LOCC 23 , Theorem 5]. This fact implies that there is no maximal element concerning LOCC even among the SLOCC orbit $\mathcal{O}_{S L O C C}\left(\sum_{i=1}^{d}|i i i\rangle\right)$.

In order to treat the multipartite extension of maximally entangled states, we introduce a new class of multipartite pure states. A multipartite pure state $|\psi\rangle$ is reduced separable when there exists a party, concerning which, the partial trace of $|\psi\rangle$ is fully separable, namely the convex sum of fully factorized states $\bigotimes_{i}\left|a_{i}\right\rangle\left\langle a_{i}\right|$. In other words a reduced separable pure state is the purification of a fully separable state. To characterize the latter, we can use the cardinality $c(\rho)$ which is defined as the minimal number of product pure components required to construct the fully separable state $\rho[24,25]$

$$
\rho=\sum_{i=1}^{c(\rho)} p_{i}\left|a_{i}^{(1)}, a_{i}^{(2)}, \ldots, a_{i}^{(N-1)}\right\rangle\left\langle a_{i}^{(1)}, a_{i}^{(2)}, \ldots, a_{i}^{(N-1)}\right| .
$$

Let its purification be the state $|\rho\rangle$ such that $\operatorname{Tr}_{N}|\rho\rangle\langle\rho|=$ $\rho$. For convenience, we also call the above number the cardinality of the purification, i.e., $c(\rho)=c(|\rho\rangle)$. Note that the tensor rank of the GHZ state coincides with its cardinality, we obtain the following theorem.

Theorem 17 In the $N$-partite system, the GHZ state with rank $d$ can generate any reduced separable pure state with the cardinality not bigger thand by LOCC. That is, the GHZ state with rank $d$ is maximal concerning LOCC among reduced separable pure states with the largest cardinality d, i.e.,

$$
\left|\mathrm{GHZ}_{d}\right\rangle \succ_{L O C C} \sum_{i=1}^{d} \sqrt{p_{i}}\left|a_{i}^{(1)}, a_{i}^{(2)}, \ldots, a_{i}^{(N-1)}, i\right\rangle .
$$

Proof. First by using the complete POVM $\left\{\sum_{j=1}^{d} \sqrt{p_{j}}|j\rangle\langle j \oplus k|\right\}, k=1, \cdots, d$ and local unitary operations, we can realize $\left|\mathrm{GHZ}_{d}\right\rangle \succ_{\text {LOCC }} \sum_{i=1}^{d} \sqrt{p_{i}}|i\rangle^{\otimes N}$. Second we consider the complete POVM on system $A_{1}$ such that

$$
P_{k}=\sum_{j=1}^{d} e^{\frac{2 \pi I}{d} j k}\left|a_{j}^{(1)}\right\rangle\langle j|
$$

, which satisfy $\sum_{k=1}^{d} P_{k}^{\dagger} P_{k}=I$. Then we get always one of the following states

$$
\sum_{j=1}^{d} e^{\frac{2 \pi I}{d} j k} \sqrt{p_{j}}\left|a_{j}^{(1)}\right\rangle|j\rangle^{\otimes(N-2)}|j\rangle, k=1, \cdots, N .
$$

Starting from this state, we perform the complete POVM $\left\{\sum_{j=1}^{d} e^{\frac{2 \pi I}{d} j k}\left|a_{j}^{(2)}\right\rangle\langle j|\right\}$ on system $A_{2}$, to get the state $\sum_{j=1}^{d} e^{I \theta_{j}} \sqrt{p_{j}}\left|a_{j}^{(1)}, a_{j}^{(2)}\right\rangle|j\rangle^{\otimes(N-3)}|j\rangle$. By using the iterative method we can finally get the state

$$
\sum_{i=1}^{d} e^{I \theta_{j}^{\prime}} \sqrt{p_{i}}\left|a_{i}^{(1)}, a_{i}^{(2)}, \ldots, a_{i}^{(N-1)}, i\right\rangle
$$

which can be recovered to the asserted state via a phase gate on system $A_{N}$. This completes the proof.

One may similarly get the LOCC partial order for more tripartite states, e.g., to transform $\left|\mathrm{GHZ}_{d}\right\rangle$ into $\sum_{i=1}^{d}\left|a_{i}, b_{i}, c_{i}\right\rangle$. Note that the partial order is usually non-invertible, because two states are LOCCinterconvertible if and only if they are unitarily equivalent [4]. 


\section{CONCLUSIONS}

In this paper, we have built the concepts of MCLOCC and MCSLOCC and showed their relations to the LOCC and SLOCC environment. These environments form the criteria in our classification hierarchy for multipartite states. In particular, We have classified the SLOCCequivlant states by using tensor rank which is a basic concept from algebraic complexity. Besides, we have de- rived the SLOCC and LOCC partial order of different sets and orbits from the hierarchy. Our method is essentially different from the previous ones in literature, which relies only on the local ranks. The results are important for understanding the convertibility between multipartite states. A further direction from this paper is to characterize the conversion between states with identical tensor rank under LOCC. This may give a better interpretation of tensor rank for studying multipartite states.
[1] C. H. Bennett, G. Brassard, C. Crepeau, R. Jozsa, A. Peres, and W. Wootters, Phys. Rev. Lett. 70, 1895 (1993).

[2] H. J. Briegel and R. Raussendorf Phys. Rev. Lett. 86, 910 (2001).

[3] C. H. Bennett, H. J. Bernstein, S. Popescu, and B. Schumacher, Phys. Rev. A 53, 2046 (1996).

[4] Bennett C H, Popescu S, Rohrlich D, Smolin J A, and Thapliyal A V, Phys. Rev. A 63, 012307 (2000).

[5] W. Dür, G. Vidal, and J. I. Cirac, Phys. Rev. A 62, 062314 (2000).

[6] D. M. Greenberger, M. Horne, and A. Zeilinger, Bell's Theorem, Quantum theory and Conceptions of the Universe, edited by M. Kafatos (Kluwer, 1989), p.69.

[7] A. Miyake, Phys. Rev. A 67, 012108 (2003).

[8] Lin Chen, Yi-Xin Chen and Yu-Xue Mei, Phys. Rev. A 74, 052331 (2006).

[9] E. Chitambar, C. A. Miller, and Yaoyun Shi, J. Math. Phys.51, 072205 (2010).

[10] C. H. Bennett, D. DiVincenzo, J. Smolin, and W. Wootters, Phys. Rev. A 54, 3824 (1996).

[11] Note that this is different from the previous concept of multi-copy transformation, which requires that the input and output states have identical number of copies, i.e., $|\varphi\rangle^{\otimes n} \rightarrow|\varphi\rangle^{\otimes n}$. In particular, the nonasymptotic case has been handled in the paper, S. Bandyopadhyay, V. Roychowdhury, and U. Sen, Phys. Rev. A 65, 052315
(2002).

[12] M. Nielsen, Phys. Rev. Lett. 83, 436 (1999).

[13] J. Eisert and H. J. Briegel, Phys. Rev. A 64, 022306 (2001).

[14] Runyao Duan, Yaoyun Shi, quant-ph/0911.0879 (2009).

[15] E. Chitambar, R. Duan, and Y. Shi, Phys. Rev. Lett. 101, 140502 (2008)

[16] Lin Chen, Eric Chitambar, Runyao Duan, Zhengfeng Ji, and Andreas Winter, quant-ph/1003.3059.

[17] Eric Chitambar, Runyao Duan, and Yaoyun Shi, Phys. Rev. A 81, 052310 (2010).

[18] T. Bastin, S. Krins, P. Mathonet, M. Godefroid, L. Lamata, and E. Solano, Phys. Rev. Lett. 103, 070503 (2009).

[19] J. B. Kruskal, Lin. Alg. Appl. 18, 95 (1977).

[20] P. Bürgisser, M. Clausen and M.A. Shokrollahi, Algebraic Complexity Theory, Springer-Verlag, Berlin, 1997.

[21] J. Haastad, J. Algorithms 11, 644 (1990).

[22] J. Ja' Ja', STOC' 78: Proceedings of the Tenth Annual ACM Symposium on Theory of Computing (ACM, New York, 1978), p. 173.

[23] S. Turgut, Y. Gul, and N. K. Pak, Phys. Rev. A 81, 012317 (2010).

[24] R. Lockhart, J. Math. Phys. 41, 6766 (2000).

[25] Erik Alfsen and Fred Shultz, J. Math. Phys.51, 052201 (2010). 